\title{
A Prosaic Explanation for the Anomalous Accelerations Seen in Distant Spacecraft
}

\author{
Edward M. Murphy日 \\ The Johns Hopkins University \\ Dept. of Physics and Astronomy \\ 3400 N. Charles Street \\ Baltimore, MD 21218
}

(September 21, 1998)

\begin{abstract}
Anderson, et al. 6 have recently reported the discovery of an apparent anomalous, weak, long-range acceleration in the Pioneer 10/11 and Ulysses spacecraft. I believe that this result can be explained by non-isotropic radiative cooling of the spacecraft electronics through passive radiators on the spacecraft surface. These radiators are preferentially placed on the anti-solar side of the spacecraft to avoid heating by solar radiation. The power transmitted through these radiator panels can explain the observed acceleration within the observational errors.
\end{abstract}

PACS numbers: 04.80.-y,95.10.Eg,95.55.Pe

\section{INTRODUCTION}

Anderson, et al. [1] have recently modeled the accelerations acting on the Pioneer 10, Pioneer 11, Ulysses, and Galileo spacecraft. They find an anomalous, excess acceleration of $8.5 \times 10^{-8} \mathrm{~cm} \mathrm{~s}^{-2}$ directed towards the Sun. They have ruled out excess gravitational forces due to the Galaxy and unidentified planetesimals, errors in the orbital and rotational parameters of the Earth, spacecraft gas leaks, and errors in the planetary ephemeris as explanations for the acceleration. In addition, the authors rule out radiation pressure from thermal radiation generated by the spacecraft radioisotope thermoelectric generators (RTGs). Anderson et al. assume that the thermal radiation generated by the RTGs is isotropically radiated and results in no net force on the spacecraft. However, I believe that this assumption overlooks the fact that the electrical energy produced by the RTGs is dissipated in a non-isotropic manner.

Spacecraft designed to travel beyond the inner solar system cannot rely on currently available solar cells to provide power, as the size of the solar arrays would be prohibitively large. Therefore, missions to the outer solar system have used RTGs to provide power. RTGs rely on the thermal energy generated by the radioactive decay of $\mathrm{Pu}^{238}$ to heat a semiconductor junction which generates an electrical current. RTGs have an electrical conversion efficiency of a few percent [2]. For example, the Ulysses RTGs generate $4500 \mathrm{~W}$ of thermal power and produce $280 \mathrm{~W}$ of electrical power (at the beginning of the mission). The available power decreases with time due to degradation in the semiconductor junction and, to a much lesser degree, the decay of the $\mathrm{Pu}^{238}$ [2].

The excess thermal power generated by the RTGs is dumped radiatively by cooling fins located on the outer surface of the cylindrical RTG structure (this is true of all the spacecraft considered here). The geometry of the fins is complex and the thermal radiation dissipated from the surface will not be isotropic. However, a cursory examination of the Pioneer and Ulysses RTG designs shows that they are cylindrically symmetric. Even though it is not isotropic, the escaping radiation will not impart a net force on the spacecraft since it is dissipated symmetrically.

The same is not true of the electrical energy created by the RTGs. The electrical energy is transported to a main power bus from where it is distributed to the individual subsystems of the satellite to provide power for operating the electronics. These electronics are typically found in a single large electronics bay (which contains most of the essential systems) and science instruments distributed throughout and around the spacecraft. To prevent the electronics from overheating, the waste heat dissipated by the electronics is radiated from the spacecraft by surface radiators. In most cases, radiators are located on the anti-solar side of the spacecraft to prevent the panels from being heated by solar radiation. Because the radiator panels are preferentially located on the antisolar side of the spacecraft, their radiation will cause an acceleration of the spacecraft toward the Sun.

From conservation of momentum arguments, it is easy to show that the acceleration, $a_{P}$, produced by an amount of radiated power, $P$, is $a_{P}=P(m c)^{-1}$ where $m$ is the mass of the spacecraft and $c$ is the speed of light. This assumes that the radiated power is tightly collimated (i.e. it carries all the momentum in a single direction). In fact, however, the radiation from a flat plate is spread over $2 \pi$ steradians. In the case of a flat Lambertian source (i.e. one in which the intensity is independent of viewing angle [3]), the momentum carried away perpendicular to the plate surface will be $2 / 3$ of the total. 


\section{ULYSSES}

The Ulysses spacecraft is spin stabilized with the rotation axis pointing approximately toward the Earth (and the Sun when the spacecraft is near aphelion). The antiEarth (anti-solar) side is always in the spacecraft shadow. The majority of the electrical components in the Ulysses spacecraft are located in a single thermal enclosure [4]. The waste heat from the electronics is radiated through a large, flat radiator panel on the anti-solar side of the spacecraft. The interior electronics radiate their heat to the panel, which in turn radiates the heat into space. In addition, the traveling wave tube amplifier (which dissipates $43 \mathrm{~W}$ alone) is directly thermally coupled to the surface radiator. Except for the anti-solar side, the spacecraft is covered in multi-layer insulation (MLI) blankets. A large 1.65 meter diameter antenna covers most of the Earth facing side.

A power budget for the Ulysses spacecraft for January 1998 [4] indicates that Ulysses' systems are drawing $231 \pm$ $3 \mathrm{~W}$ of electrical power. Of this, I calculate that $27 \pm$ $10 \mathrm{~W}$ is dissipated by scientific instruments and heaters outside the main thermal enclosure and another $20 \mathrm{~W}$ is radiated by the transmitter. Therefore, in a steady state, the main thermal enclosure must radiate $184 \pm 13 \mathrm{~W}$ of power. Because the error estimates are systematic, rather than statistical, I have added them directly rather than in quadrature. Some fraction of this power will escape through the MLI thermal blankets and the remainder will be radiated through the large surface radiator on the anti-solar side of the spacecraft. MLI blankets typically radiate $8 \mathrm{~W} \mathrm{~m}^{-2}$ [2] into space. Only the blankets on the side of the spacecraft will radiate internal heat because the solar facing blankets of Ulysses allow a net input of heat into the thermal enclosure due to solar heating, though this input power is small $(\sim 2 \mathrm{~W})$ compared to electrical power when the spacecraft is at aphelion. When near perihelion, Ulysses compensates for the excess input solar heating by dumping excess electrical energy into resistors on the outside of the spacecraft. About $50 \mathrm{~W}$ of excess electrical power is dumped when the spacecraft is near perihelion [4].

I calculate that there are $3.0 \pm 1.0 \mathrm{~m}^{2}$ of MLI blankets on the sides of the main thermal enclosure of Ulysses resulting in $24 \pm 8 \mathrm{~W}$ escaping through the MLI blankets. This implies that the total power radiated through the spacecraft radiator on the anti-solar side of Ulysses is $(160 \pm 21) \mathrm{W}$. The acceleration produced by this power is $a_{P}=(10.3 \pm 1.3) \times 10^{-8} \mathrm{~cm} \mathrm{~s}^{-2}$ assuming a spacecraft mass of $345 \mathrm{~kg}$ and that the radiator is a Lambertian source $(2 / 3$ of the momentum is carried away perpendicular to the radiator). Since the radiator faces away from the Sun, the direction of this acceleration is toward the Sun. This matches, to within the errors, the anomalous acceleration reported by Anderson et al. [1] for Ulysses of $a_{P}=(12 \pm 3) \times 10^{-8} \mathrm{~cm} \mathrm{~s}^{-2}$.

\section{PIONEER 10 AND 11}

Toward the ends of their missions, the Pioneer 10/11 spacecraft were drawing $80 \mathrm{~W}$ of electrical power [1] from their RTGs, which was sufficient to power the essential spacecraft systems and possibly one or two scientific instruments. Of this, $9 \mathrm{~W}$ is transmitted as RF power [1]. The essential electrical systems are located in a cylindrical hub beneath the high-gain antenna. The waste heat generated by the electronics is radiated from a series of fins on the anti-solar side of the spacecraft. Since the majority of the science instruments have been turned off, essentially all of the $71 \mathrm{~W}$ of internally dissipated electrical power is radiated from the fins. Solar heating is negligible at the Pioneers' distance from the Sun. Assuming that the current mass of Pioneer $10 / 11$ is $250 \mathrm{~kg}$, the radiated power generates an $a_{P}=6.3 \times 10^{-8} \mathrm{~cm} \mathrm{~s}^{-2}$ again assuming a Lambertian source. However, the radiator fins of the back of the Pioneer spacecraft are highly non-Lambertian sources. In fact, the fins are likely to collimate the outgoing radiation to a significant degree. If the radiation were fully collimated, the resulting acceleration would be $a_{P}=9.5 \times 10^{-8} \mathrm{~cm} \mathrm{~s}^{-2}$. The actual value is likely to lie between these extremes. These estimates of the acceleration due to radiative cooling closely match the $a_{P}=8.5 \times 10^{-8} \mathrm{~cm} \mathrm{~s}^{-2}$ reported by Anderson, et al. 11] for the Pioneer 10 and 11 spacecraft.

The essential electrical systems must remain powered at all times. Although the thermal power output of the RTGs is expected to decrease with time, the power drawn by the essential spacecraft electronics is nearly constant and, therefore, the acceleration imparted by the thermal radiation from the spacecraft radiators should also be constant with time. As the missions have progressed, various science instruments have been turned on and off. Most of these instruments are located on the periphery of the spacecraft, or on long booms reaching 3 meters or more from the spacecraft. These instruments are, typically, small enough that they radiate their power isotropically and do not employ a radiator system. Therefore, as the the science instruments are cycled on and off, there should be little effect on the net acceleration.

\section{CONCLUSIONS}

I have shown that the most likely explanation for the anomalous accelerations found by Anderson et al. in the Pioneer 10/11 and Ulysses spacecraft is radiation pressure from spacecraft radiators which prevent the buildup of heat in the electronics systems. A more detailed analysis of the thermal designs of the spacecraft, including an examination of their power requirements 
with time, would allow us to reduce the associated errors and would make it possible to search for additional accelerations in the Anderson et al. data at a level of $a_{P}=1-2 \times 10^{-8} \mathrm{~cm} \mathrm{~s}^{-2}$.

\section{ACKNOWLEDGMENTS}

I wish to thank Shaun Standley of the Ulysses Project at JPL for very informative conversations concerning the Ulysses thermal design and power budget. I also wish to thank Scott Friedman and Alexandra Cha for helpful conversations. This work was supported by NASA contract NAS5-32985 (FUSE).

* Electronic address: emurphy@pha.jhu.edu.

[1] J. D. Anderson, P. A. Laing, E. L. Lau, A. S. Liu, M. M. Nieto, and S. G. Turyshev, Phys. Rev. Lett., in press (1998), gr-qc/9808081.

[2] V. L. Piscane and R. C. Moore, Fundamentals of Space Systems, (Oxford University Press, New York, 1994).

[3] R. W. Boyd, Radiometry and the Detection of Optical Radiation, (John Wiley \& Sons, New York, 1983).

[4] S. Standley, private communication (1998). 